\title{
Biased diffusion in confined media: Test of the Fick-Jacobs approximation and validity criteria
}

\author{
P. S. Burada, ${ }^{1}$ G. Schmid, ${ }^{1}$ D. Reguera, ${ }^{2}$ J. M. Rubí, ${ }^{2}$ and P. Hänggi ${ }^{1}$ \\ ${ }^{1}$ Institut für Physik, Universität Augsburg, Universitätsstrasse 1, D-86135 Augsburg, Germany \\ ${ }^{2}$ Departament de Física Fonamental, Facultat de Física, Universidad de Barcelona, Diagonal 647, E-08028 Barcelona, Spain
}

(Received 24 January 2007; published 15 May 2007)

\begin{abstract}
We study biased, diffusive transport of Brownian particles through narrow, spatially periodic structures in which the motion is constrained in lateral directions. The problem is analyzed under the perspective of the Fick-Jacobs equation, which accounts for the effect of the lateral confinement by introducing an entropic barrier in a one-dimensional diffusion. The validity of this approximation, based on the assumption of an instantaneous equilibration of the particle distribution in the cross section of the structure, is analyzed by comparing the different time scales that characterize the problem. A validity criterion is established in terms of the shape of the structure and of the applied force. It is analytically corroborated and verified by numerical simulations that the critical value of the force up to which this description holds true scales as the square of the periodicity of the structure. The criterion can be visualized by means of a diagram representing the regions where the Fick-Jacobs description becomes inaccurate in terms of the scaled force versus the periodicity of the structure.
\end{abstract}

DOI: 10.1103/PhysRevE.75.051111

PACS number(s): 05.60.Cd, 05.40.Jc, 02.50.Ey

\section{INTRODUCTION}

In many transport phenomena, such as those taking place in biological cells, ion channels, nanoporous materials and microfluidic devices etched with grooves and chambers, Brownian particles, instead of diffusing freely in the host liquid phase, undergo a constrained motion. The uneven shape of these structures regulates the transport of particles yielding important effects exhibiting peculiar properties. The results have implications in processes such as catalysis, osmosis, and particle separation [1-9], and for the noiseinduced transport in periodic potential landscapes that lack reflection symmetry (ratchet systems) [10-12] or ratchet transport mechanisms that are based on asymmetric geometries, termed "entropic" ratchet devices [10-13]. For example, it has been found that the separation of DNA fragments in narrow channels [14-16] is largely influenced by their shape. The translocation of structured polynucleotides through nanopores also allows one to determine their sequence and structure [17-19].

The motion of the particles through these quasi-onedimensional structures can in principle be analyzed by means of the standard protocol of solving the Smoluchowski equation with the appropriate boundary conditions imposed. Whereas this method has been very successful when the boundaries of the system possess a regular shape, the challenge to solve the boundary value problem in the case of uneven boundaries represents typically a very difficult task. A way to circumvent this difficulty consists in coarsening the description by reducing the dimensionality of the system, keeping only the main direction of transport, but taking into account the irregular nature of these boundaries by means of an entropic potential. The resulting kinetic equation for the probability distribution, the Fick-Jacobs (FJ) equation, is similar in form to the Smoluchowski equation, but now contains an entropic term. The entropic nature of this term leads to a genuine dynamics which is very different from that observed when the potential has an energetic origin [20]. It has been shown that the FJ equation can provide a very accurate description of entropic transport in two- (2D) and three-dimensional (3D) channels of varying cross section $[20,21]$.

However, the derivation of the FJ equation entails a tacit approximation: The particle distribution in the transverse direction is assumed to equilibrate much faster than that in the main (unconstrained) direction of transport. This equilibration justifies the coarsening of the description, leading in turn to a simplification of the dynamics, but raises a question about its validity when an external force is applied. To establish the validity criterion of a FJ description for such biased diffusion in confined media is, due to the ubiquity of this situation, a subject of primary importance.

Our objective with this work is to investigate in greater detail the FJ approximation for biased diffusion and to set up a corresponding criterion describing its regime of validity. We will analyze the biased movement of Brownian particles in $2 \mathrm{D}$ and $3 \mathrm{D}$ periodic channels of varying cross section and formulate different criteria for the validity of such a FJ description.

The paper is organized in the following way. In Sec. II, we describe the physical situation and introduce the model defined through the corresponding Langevin and FokkerPlanck equations. In Sec. III, we introduce the FJ approach for the unbiased situation and extend it to the driven case. Section IV is devoted to establishing a criterion for the validity of the FJ approximation derived by comparing the different characteristic time scales of the process. In Sec. V, the accuracy of the FJ description is tested against numerical simulations for a 2D periodic channel, and the conditions of validity are summarized in a diagram in terms of the scaled force versus the periodicity of the structure. In Sec. VI, we provide further explanations on, when and why the equilibration assumption fails, and when the FJ approach leads to usable results. Finally, in Sec. VII we present our main conclusions. 


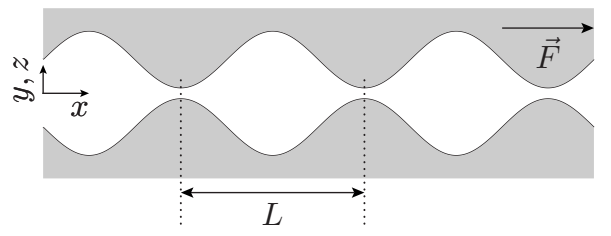

FIG. 1. Schematic diagram of the channel confining the motion of the biased Brownian particles. The half-width $\omega$ is a periodic function of $x$ with periodicity $L$.

\section{DIFFUSION IN CONFINED SYSTEMS}

In typical transport processes through pores or channels (like the one depicted in Fig. 1), the motion of the suspended particles is induced by application of an external potential $V(\vec{r})$ resulting in a force $\vec{F}$. In general, the dynamics of the suspended Brownian particles is governed by Langevin's equation

$$
m \ddot{\vec{r}}(t)=-\eta \dot{\vec{r}}(t)-\vec{\nabla} V(\vec{r}(t))+\sqrt{\eta k_{\mathrm{B}} T} \vec{\xi}(t),
$$

where $\vec{r}$ is the two- or three-dimensional position vector of a particle of mass $m, \eta$ is its friction coefficient, $k_{\mathrm{B}}$ is the Boltzmann constant, $T$ is the temperature, and a dot over the quantity refers to a time derivative. The thermal fluctuations due to the coupling of the particle with the environment are modeled by a zero-mean Gaussian white noise $\vec{\xi}(t)$, obeying the fluctuation-dissipation relation $\left\langle\xi_{i}(t) \xi_{j}\left(t^{\prime}\right)\right\rangle=2 \delta_{i j} \delta\left(t-t^{\prime}\right)$ for $i, j=x, y, z$. In the overdamped case, i.e., when $m \ddot{\vec{r}}(t) \ll \dot{\vec{r}}(t)$, the inertia term in Eq. (1) can be safely neglected and the Langevin equation describing the dynamics of a Brownian particle within the channel reads

$$
\eta \frac{d \vec{r}}{d t}=-\vec{\nabla} V(\vec{r}(t))+\sqrt{\eta k_{\mathrm{B}} T} \vec{\xi}(t)
$$

In addition to Eq. (2), the full problem is set up by imposing reflecting boundary conditions at the channel walls.

The corresponding Fokker-Plank equation for the time evolution of the probability distribution $P(\vec{r}, t)$ takes the form $[22,23]$

$$
\frac{\partial P(\vec{r}, t)}{\partial t}=-\vec{\nabla} \cdot \vec{J}(\vec{r}, t)
$$

where $\vec{J}(\vec{r}, t)$ is the probability current,

$$
\vec{J}(\vec{r}, t)=-\left(\frac{\vec{\nabla} V(\vec{r})}{\eta}+D_{0} \vec{\nabla}\right) P(\vec{r}, t)
$$

and

$$
D_{0}=k_{\mathrm{B}} T / \eta
$$

denotes the diffusion coefficient of the suspended particles.

Due to the impenetrability of the channel walls, the normal component of the probability current $\vec{J}(\vec{r}, t)$ vanishes at the boundaries. If $\vec{n}$ denotes the vector perpendicular to the channel walls, the reflecting boundary conditions read

$$
\vec{J}(\vec{r}, t) \cdot \vec{n}=0
$$

In this paper we focus on the case of a symmetric 2D channel where the force $F$ is constant and directed along the axis (cf. Fig. 1). The half-width of the 2D channel is given by a periodic function $\omega(x)$, i.e., $\omega(x+L)=\omega(x)$ for all $x$. In this case, the boundary condition reads

$$
-\frac{d \omega(x)}{d x}\left(\frac{F}{\eta} P(x, y, t)-D_{0} \frac{\partial P(x, y, t)}{\partial x}\right)-D_{0} \frac{\partial P(x, y, t)}{\partial y}=0
$$

at $y= \pm \omega(x)$. For an arbitrary form of $\omega(x)$, the boundary value problem defined through Eqs. (3), (4), and (7) is very difficult to solve. Despite the inherent complexity of this problem an approximate solution can be found by introducing an effective one-dimensional description where geometric constraints and bottlenecks are considered as entropic barriers $[9,20,21,24-26]$.

\section{FICK-JACOBS APPROXIMATION}

In the absence of an external force, i.e., when $F=0$, it was shown $[24,25]$ that the dynamics of particles in confined structures (such as that of Fig. 1) can be described by the FJ equation

$$
\frac{\partial P(x, t)}{\partial t}=\frac{\partial}{\partial x}\left(D_{0} h(x) \frac{\partial}{\partial x} \frac{P}{h(x)}\right)
$$

obtained from the 3D (or 2D) Smoluchowski equation after elimination of the $y$ and $z$ coordinates. Here $P(x, t)$ is the probability distribution function along the axis of the $2 \mathrm{D}$ or 3D channel, $h(x)$ is the dimensionless transverse cross section $h(x):=\pi[\omega(x) / L]^{2}$ in $3 \mathrm{D}$, and the dimensionless width $h(x):=2 \omega(x) / L$ in $2 \mathrm{D}$, where $\omega(x)$ is the radius of the channel in 3D (or the half-width of the channel in 2D). This description is valid for $\left|\omega^{\prime}(x)\right| \ll 1$ [the prime refers to the first derivative, i.e., $\left.\omega^{\prime}(x)=d \omega(x) / d x\right]$, and it has been shown that the introduction of an effective $x$-dependent diffusion coefficient can considerably improve the accuracy of the kinetic equation, thus extending its validity to more winding structures $[21,25,26]$. The expression

$$
D(x)=\frac{D_{0}}{\left[1+\omega^{\prime}(x)^{2}\right]^{\alpha}},
$$

where $\alpha=1 / 3,1 / 2$ for two and three dimensions, respectively, has been shown to accurately account for the curvature effects [21].

In the presence of a constant force $F$ along the direction of the channel the FJ equation can be recast into the expression $[20,21]$

$$
\frac{\partial P}{\partial t}=\frac{\partial}{\partial x}\left(D(x) \frac{\partial P}{\partial x}+\frac{D(x)}{k_{\mathrm{B}} T} \frac{\partial A(x)}{\partial x} P\right),
$$

which defines the free energy $A(x):=E-T S$ $=-F x-T k_{\mathrm{B}} \ln h(x)$, where $E=V=-F x$ denotes the energy contribution and $S=k_{\mathrm{B}} \ln h(x)$ the entropy contribution. For a symmetric channel with periodicity $L$, the free energy assumes the form of a periodic tilted potential. 
Note that Eq. (10) may typically describe the time evolution of a 1D particle distribution within an energy landscape. In the present context, however, due to the reduction of the geometric confinements in 2D or 3D space into one dimension, we end up with an entropic contribution to the free energy. In absence of a fixed bias $F=0$, we deal with a pure entropic situation with the free energy reading $A(x)=-T k_{\mathrm{B}} \ln h(x)$. Equation (10) then corresponds to a FJ equation with a spatial dependent diffusion coefficient. Likewise, for a situation involving solely transport in a onedimensional energy landscape, the free energy reduces to $A(x)=V(x)$ with $d \omega(x) / d x=0$, yielding to the well-known Fokker-Planck equation in 1D, with a constant diffusion coefficient $D_{0}$, reading

$$
\frac{\partial P}{\partial t}=\frac{\partial}{\partial x}\left(\frac{V^{\prime}}{\eta} P+D_{0} \frac{\partial P}{\partial x}\right) .
$$

Recently, we have shown that the dynamics of a confined Brownian particle, in the presence of an applied bias, can accurately be described by means of Eq. (10) [20]. In the presence of very strong applied bias, and for more winding structures, however, the FJ equation becomes inaccurate. In the present work we present further numerical and analytical results and will set up tailored criteria under which the FJ approximation assumes good validity.

\section{Particle current}

One of the key quantities in transport through quasi-onedimensional structures is the study of the average particle current $\langle\dot{x}\rangle$, or equivalently the nonlinear mobility, which is defined as the ratio of the average particle current and the applied force $F$. For the average particle current we derive an expression that is similar to the Stratonovich formula for the current in titled periodic energy landscapes [27-30], but with a spatial diffusion coefficient. A detailed derivation of this expression is given in the Appendix [see Eq. (A12)]. Hence, we obtain the nonlinear mobility for a $2 \mathrm{D}$ channel with a shape defined by $\omega(x)$ :

$$
\mu(F, L, \beta) \eta:=\frac{\langle\dot{x}\rangle}{F} \eta=\frac{1}{\beta F} \frac{1-\exp (-\beta F L)}{\int_{0}^{L} \frac{d x}{L} e^{-\beta A(x)} \int_{x}^{x+L} d x^{\prime}\left[1+\left(\frac{d \omega\left(x^{\prime}\right)}{d x^{\prime}}\right)^{2}\right]^{\alpha} e^{\beta A\left(x^{\prime}\right)}}
$$

where we have made use of Eq. (9) and the relations $D_{0}$ $=k_{\mathrm{B}} T / \eta$ and $\beta=1 / k_{\mathrm{B}} T$. Substituting $z^{\prime}=x^{\prime} / L$ and $z=x / L$, the nonlinear mobility scaled with the friction coefficient $\eta$ can be expressed, for the case of constant forcing, in terms of a single, dimensionless scaling parameter [20]

$$
f:=\beta F L .
$$

Therefore, Eq. (12) for $A(x)=-F x-\beta^{-1} \ln h(x)$ leads to

$$
\mu(f) \eta=\frac{1}{f} \frac{1-\exp (-f)}{\int_{0}^{1} d z I(z, f)}
$$

where

$$
\begin{aligned}
I(z, f)= & \exp (f z) h(L z) \int_{z}^{z+1} d z^{\prime} \exp \left(-f z^{\prime}\right) h^{-1}\left(L z^{\prime}\right) \\
& \times\left[1+\left(\frac{1}{L} \frac{d \omega\left(L z^{\prime}\right)}{d z^{\prime}}\right)^{2}\right]^{\alpha} .
\end{aligned}
$$

Equation (14) can be transformed into Eq. (6) in Ref. [20] by interchanging the order of integration.

The asymptotic values of the nonlinear mobility for the cases $f \rightarrow 0$ and $f \rightarrow \infty$ can be evaluated to read

$$
\lim _{f \rightarrow 0} \mu(f) \eta=\frac{1}{\langle h(x)\rangle\left\langle\frac{D_{0}}{D(x)} h^{-1}(x)\right\rangle}
$$

and

$$
\lim _{f \rightarrow \infty} \mu(f) \eta=\frac{1}{\left\langle\frac{D_{0}}{D(x)}\right\rangle}
$$

where

$$
\langle g(x)\rangle=\frac{1}{L} \int_{0}^{L} g(x) d x
$$

is the average over a spatial period, given an arbitrary periodic function $g(x)$.

\section{VALIDITY OF THE FICK-JACOBS DESCRIPTION IN THE PRESENCE OF A CONSTANT BIAS}

The reduction of dimensionality done implicitly in the formulation of the FJ equation relies on the assumption of equilibration in the transverse direction. An estimate of the conditions under which equilibration occurs can be made by analyzing the different time scales involved in the problem. For the sake of simplicity, let us focus on the situation of a 2D channel, although the same discussion can readily be 
extended to 3D. In a 2D channel in the presence of an external force, in the axial direction, one can identify three characteristic processes, with the corresponding different time scales. One is diffusion in the transverse direction over a distance $\Delta y$, whose time scale is

$$
\tau_{y}=\frac{\Delta y^{2}}{2 D_{0}} .
$$

Similarly, the time scale associated with diffusion in the axial direction is

$$
\tau_{x}=\frac{\Delta x^{2}}{2 D_{0}} .
$$

The third time scale is defined through the characteristic time associated with the drift (ballistic motion) over a distance $\Delta x$ given by

$$
\frac{\Delta x}{\tau_{\text {drift }}} \sim \frac{F}{\eta}=\frac{f D_{0}}{L},
$$

where we have used the scaling factor $f=F L / k_{\mathrm{B}} T$. Rearranging the previous expression, we obtain

$$
\tau_{\text {drift }}=\frac{L \Delta x}{f D_{0}} .
$$

In order to have a good equilibration in the transverse direction, the characteristic time scale associated with diffusion in this direction has to be much smaller than the other two time scales. Therefore, in the absence of an external force, equilibration in the transverse direction occurs if $\tau_{y} / \tau_{x} \ll 1$. This results in the condition

$$
\frac{\Delta y^{2}}{\Delta x^{2}} \sim \omega^{\prime}(x)^{2} \ll 1,
$$

which constitutes the validity criterion of the FJ approach as put forward by Zwanzig [25].

In the presence of a force along the axis, equilibration in the transverse direction demands that the condition $\tau_{y} / \tau_{\text {drift }}$ $\ll 1$ also holds. Consequently,

$$
\frac{f \Delta y^{2}}{2 L \Delta x} \sim \frac{2 f \omega(x)^{2}}{L^{2}} \ll 1,
$$

where in the second step we have replaced the characteristic distances $\Delta y$ by the width $2 \omega(x)$ and $\Delta x$ by $L$.

A general estimate of the criterion that has to be satisfied is that $\max \left(\tau_{y} / \tau_{x}, \tau_{y} / \tau_{\text {drift }}\right) \ll 1$. An even stronger criterion in order for the FJ description to hold in the presence of a constant force can be put forward by considering the sum of the two ratios [see Eqs. (22) and (23)], i.e.,

$$
\omega^{\prime}(x)^{2}+\frac{2 f \omega(x)^{2}}{L^{2}} \ll 1 .
$$

Equation (24) provides a quite stringent criterion that indicates when the FJ description of a system is expected to be valid. Note also that this is a local criterion, i.e., for a given channel, there will be regions [associated with drastic changes in the shape of the channel, i.e., $\left.\omega^{\prime}(x)^{2} \gg 1\right]$ where equilibration in the transverse direction is not feasible, whereas in others it is fulfilled. It is then more convenient to work with a global criterion of validity rather than with a local one. One way of getting that global condition is by averaging the local criterion over the period $L$ of the channel, yielding one of our main results:

$$
\left\langle\omega^{\prime}(x)^{2}\right\rangle+\frac{2 f}{L^{2}}\left\langle\omega(x)^{2}\right\rangle \ll 1 .
$$

In order to get an explicit estimate of the dependence of the maximum force value on the periodicity $L$ of the channel, we define a critical force value $f_{c}$ for which the inequality (25) becomes an equality, i.e., $\left\langle\omega^{\prime}(x)^{2}\right\rangle+\left(2 f_{c} / L^{2}\right)\left\langle\omega(x)^{2}\right\rangle=1$. Then the critical force value reads

$$
f_{c}=\frac{L^{2}}{2\left\langle\omega(x)^{2}\right\rangle}\left[1-\left\langle\omega^{\prime}(x)^{2}\right\rangle\right],
$$

which indicates that the critical force scales asymptotically as $L^{2}$, if we fix the overall shape of the channel and change only its periodicity.

Equation (26) provides an estimate of the minimum forcing beyond which the FJ description is expected to fail in providing an accurate description of the dynamics of the system. The quantitative value of the critical force will obviously depend on the level of accuracy sought. What is really important is how it depends (or scales) with the relevant parameters of the problem.

\section{NUMERICAL SIMULATIONS FOR A 2D CHANNEL}

In order to check the consistency of the criterion proposed and the validity of the FJ description in the presence of a force, we will compare the analytical result for the scaled nonlinear mobility obtained in Eq. (14) with the simulation results of the overdamped Langevin dynamics in Eq. (2) for the 2D periodic channel sketched in Fig. 1. We remark here that the extension of our scheme to 3D with a rotational symmetry along the transport axis is possible as well. This will consume more computation time, but the overall findings remain qualitatively robust. This feature has been verified with a few numerical tests.

The shape of the channel is described by

$$
\omega(x)=a \sin (2 \pi x / L)+b,
$$

where $a$ controls the slope of the channel walls and $2(b-a)$ gives the channel width at the bottlenecks. In order to use dimensionless quantities we refer any physical length to $a$, i.e., the scaled boundary condition then reads $\omega(x)$ $=\sin (2 \pi x / L)+b$, where $b$ is now a dimensionless quantity. In our case, we have used $b=1.02$. The behavior of the quantities of interest have been corroborated by Brownian dynamic simulations performed by integration of the Langevin equation using the standard stochastic Euler algorithm. The average particle current in the $x$ direction has been derived from an ensemble average of about $3 \times 10^{4}$ trajectories according to the following expression:

$$
\langle\dot{x}\rangle=\lim _{t \rightarrow \infty}\left\langle\frac{x(t)}{t}\right\rangle .
$$




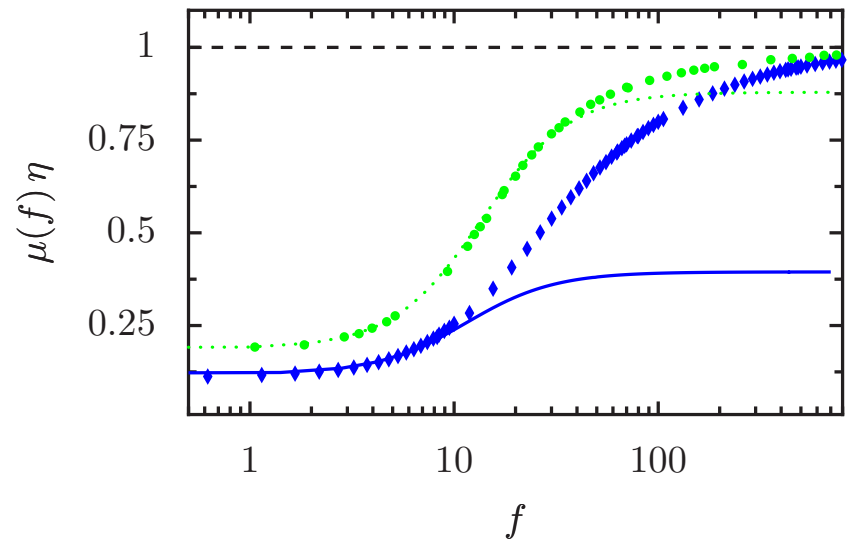

FIG. 2. (Color online) Numerically simulated (symbols) and analytically calculated [see Eq. (14)] (lines) dependence of the scaled nonlinear mobility $\mu(f) \eta$ vs the scaling parameter $f=F L / k_{\mathrm{B}} T$ for two channels in $2 \mathrm{D}$ with different spatial periods. For both channels the scaled half-width is given by $\omega(x)$ $=\sin (2 \pi x / L)+1.02 ; L=1$, diamonds and solid line (blue) $L=2 \pi$, circles and dotted line (green). The dashed line indicates the deterministic limit $\mu(f) \eta=\langle\dot{x}\rangle /(F / \eta)=1$.

In order to test the accuracy of the FJ description, we have evaluated the behavior of the nonlinear mobility as a function of the scaled force $f$, for different values of the periodicity $L$. In Fig. 2, the dependence of the scaled nonlinear mobility on $f$ is depicted for two different periodic structures of spatial period $L$, and for $b=1.02$. In both cases, the nonlinear mobility depicts a monotonic increase with increasing force. The value of the scaling parameter $f=\beta F L$ up to which the FJ approximation with space-dependent diffusion coefficient $D(x)$ provides an accurate description depends on the spatial period $L$. Consistent with the FJ approximation scheme, for rather smoothly varying cross section, or, equivalently, large periods $L$, the agreement between our precise numerics and the analytic solution is attained for considerably larger $f$ values as compared to the case with strongly winding structures or small periods. The analytic solution does not capture the correct limiting value for large $f$. Whereas the analytic result of the FJ equation tends to the value given by Eq. (16), the limiting value observed in the simulations always tends to 1 , which corroborates the deterministic limit. This indicates that, in the case of very strong forcing, the particles almost travel ballistically, and thus do not experience the effects of the boundaries. This is all consistent with the accompanying breakdown of the FJ approximation in this strong forcing limit for which the deterministic dynamics dominates the transport.

By comparing the exact numerical results with the analytic solution of the FJ equation, Eq. (14), we can identify a critical value of the scaled force, $f_{c}$, beyond which the relative error in the mobility exceeds a certain value. This critical force plays a similar role to that introduced in the previous section.

For our example of a 2D channel whose shape is defined by Eq. (27), the validity criterion given by Eq. (26) simplifies to

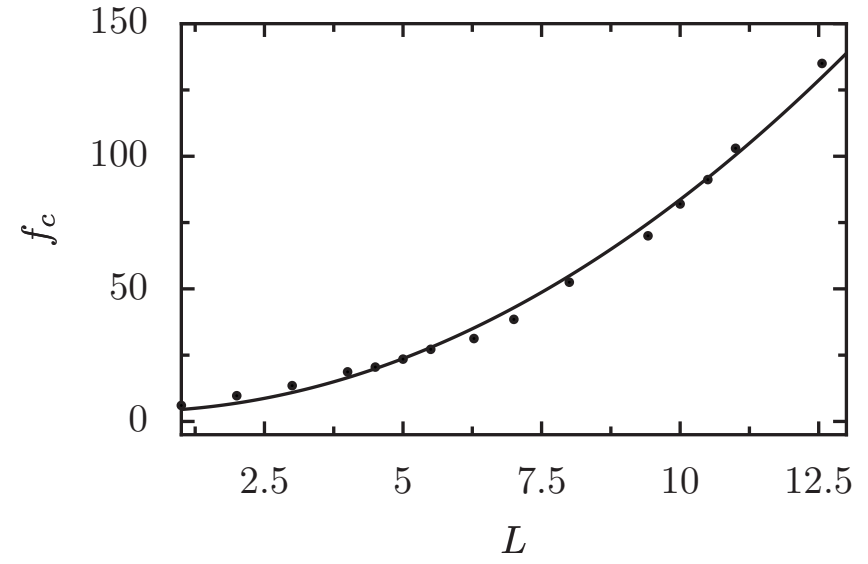

FIG. 3. Dependence of the critical value of the scaling parameter, i.e., $f_{c}$, on the periodicity $L$ of a $2 \mathrm{D}$ channel defined by the dimensionless boundary function $\omega(x)=\sin (2 \pi x / L)+1.02$. For $f<f_{c}$ the relative error of the Fick-Jacobs description is less than $1 \%$. The solid line is an $L^{2}$ fit of the critical values obtained by comparison of the approximate analytic and the exact numerical results.

$$
f_{c}=\frac{L^{2}}{1+2 b^{2}}\left(1-\frac{2 \pi^{2}}{L^{2}}\right)
$$

thus predicting that the critical value of the force scales as $L^{2}$.

This prediction has been verified by the simulations. Figure 3 shows the value of the critical force for a tolerance of $1 \%$ as a function of the periodicity $L$. The critical value of the force depends quadratically on the periodicity $L^{2}$, as predicted.

In Fig. 4 we illustrate, for the considered two-dimensional channel, the regions where the FJ approximation is accurate

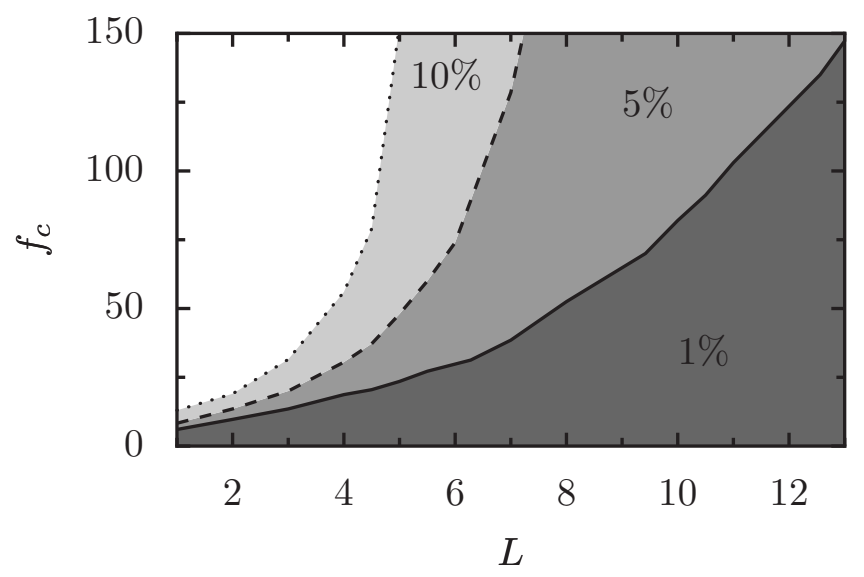

FIG. 4. Validity diagram of the Fick-Jacobs approximation for biased diffusion obtained upon a comparison between the precise numerics with the approximate analytic solution, [see Eq. (14)] for a $2 \mathrm{D}$ channel with boundary function $\omega(x)=\sin (2 \pi x / L)+1.02$. The dependence of the critical value of the scaling parameter on the periodicity is depicted for three different relative errors; $1 \%$, solid line; $5 \%$, dashed line; and $10 \%$, dotted line. Below these limiting lines the analytic treatment agrees within the corresponding prescribed relative error. 

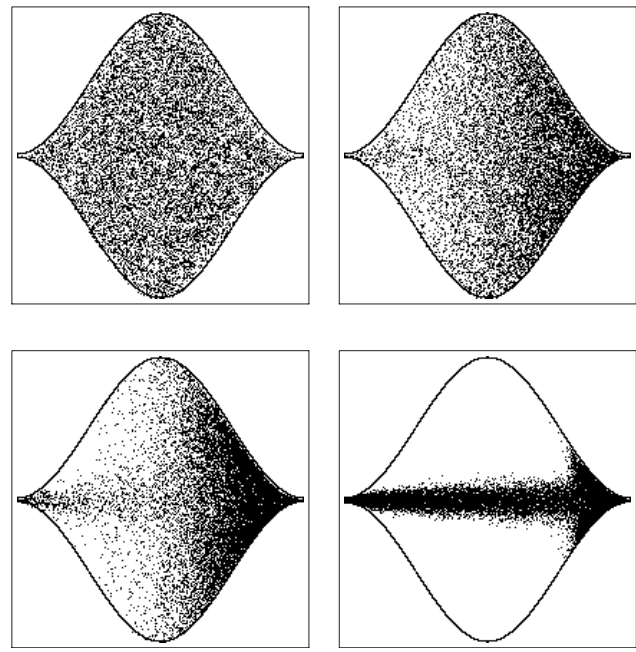

FIG. 5. Steady-state particle distribution mapped into a single period of the 2D channel defined by the boundary function $\omega(x)$ $=\sin (2 \pi x / L)+1.02$ with $L=1$ for four different values of the dimensionless scaling parameter $f$ : upper left panel, $f=0.2$; upper right panel, $f=3.0$; lower left panel, $f=7.0$; lower right panel, $f=50$.

when compared with the simulations. We depict the dependence on the periodicity $L$ of the maximal critical scaled force, obtained by comparing numerical results with the analytic solution for the particle current, for different required relative errors. This diagram shows the regions in parameter space in terms of $f$ and $L$ for which an accurate solution is provided. Thus, it is possible to provide an accurate result by using the analytic solution over a wide range of the scaled parameter and the periodicity.

\section{HYPOTHESIS OF EQUILIBRATION IN THE TRANSVERSE DIRECTION}

From our simulations, we can actually analyze the validity of the hypotheses of equilibration in the transverse direction on which the FJ description relies. In Fig. 5, we show the steady state distribution of a certain number of Brownian particles for different values of the scaled force $f$. As the force increases, we can clearly see that the particles are not

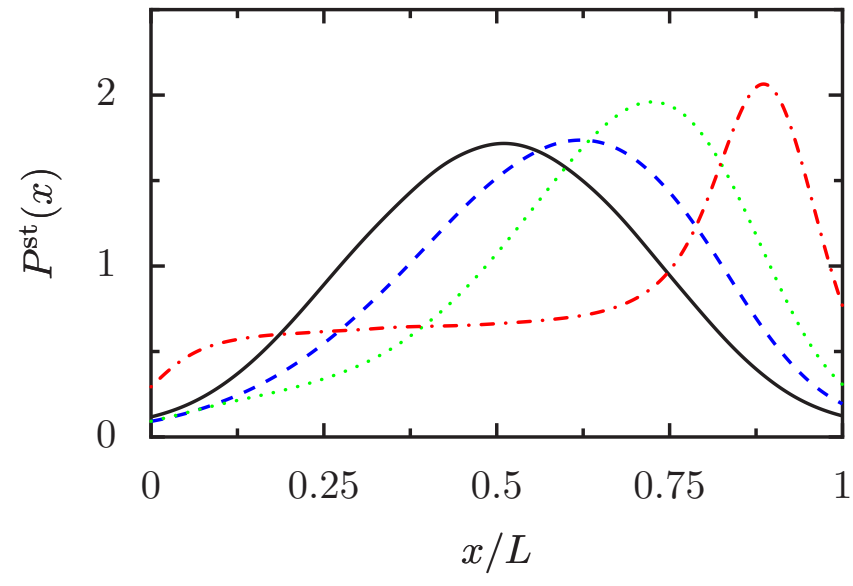

FIG. 7. (Color online) Normalized steady-state probability distribution of particles along the length of the channel $P^{\mathrm{st}}(x)$ [see Eq. (31)], for the corresponding set of scaling parameters $f$ as those chosen in Fig. 6.

homogeneously distributed in the $y$ direction, evidencing the failure of the equilibration assumption. This effect is especially dramatic in Fig. 5 (lower right panel), where the force is so strong that the particles do not fully explore the available space in the $y$ direction.

A more detailed analysis could be provided by checking the normalized steady-state probability distribution in the transverse direction at a given $x$ position, i.e.,

$$
P_{x}^{\mathrm{st}}(y):=\frac{P^{\mathrm{st}}(x, y)}{\int_{-\omega(x)}^{\omega(x)} d y P^{\mathrm{st}}(x, y)} .
$$

In Fig. 6, we represent the steady-state probability density at three different locations along the channel, corresponding to $x / L=0.2,0.5$, and 0.8 . For small values of $f$, at $x / L=0.5$, where $\omega^{\prime}(x)=0$, the $P_{x}^{\mathrm{st}}(y)$ is flat, indicating a perfect equilibration in the transverse direction. However, at $x / L=0.2$, and 0.8 , the system is not equilibrated and $P_{x}^{\text {st }}(y)$ is bell shaped. Notice that, for $x / L=0.2$ and 0.8 , the distributions are very similar for small values of $f$, corroborating that the equilibration depends on $\omega^{\prime}(x)^{2}$, which is the same in both cases.
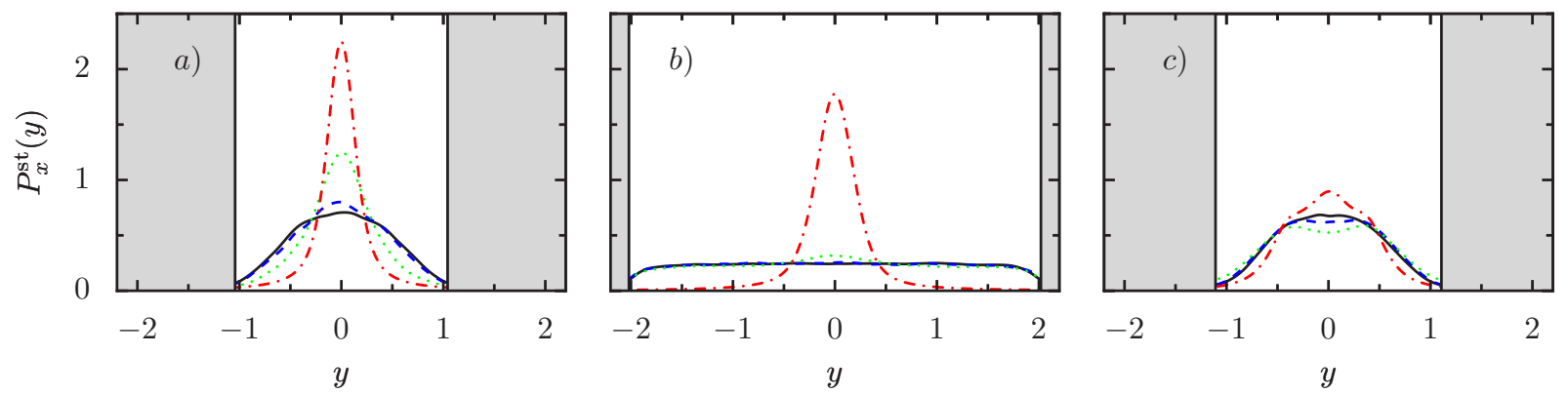

FIG. 6. (Color online) Normalized steady-state probability distribution of particles in the $y$ direction $P_{x}^{\text {st }}(y)$ [see Eq. (30)] for different values of the scaling parameter $f$ : solid line (black), $f=0.2$; dashed line (blue), $f=3.0$; dotted line (green), $f=7.0$; dash-dotted line (red), $f=50.0$, at various positions along the length of the channel with the boundary function $\omega(x)=\sin (2 \pi x / L)+1.02, L=1 ; x / L=0.2$ (a), 0.5 (b), and $0.8(\mathrm{c})$. The gray regions indicate the outside of the channel. 
At large force strengths, particles concentrate along the axis of the channel. In this situation, the assumption of equilibration along the transverse direction fails. Particles will experience the presence of the boundaries only when they are close to the bottlenecks. Hence, in the limit of very large force values, the influence of the entropic barriers practically disappears. In this limit, the correction in the diffusion coefficient leading to a spatial dependency, i.e., $D(x)$, overestimates the influence of the entropic barriers.

Figure 7 depicts the normalized stationary distribution function of particles in the $x$ direction at various force strengths, obtained from numerical simulations:

$$
P^{\mathrm{st}}(x):=\frac{\int_{-\omega(x)}^{\omega(x)} d y P^{\mathrm{st}}(x, y)}{\int_{0}^{L} d x \int_{-\omega(x)}^{\omega(x)} d y P^{\mathrm{st}}(x, y)} .
$$

At very low force strengths the particles are evenly distributed, and the probability distribution $P^{\mathrm{st}}(x)$ scales with the cross section of the channel. On increasing the force, the maximum of $P^{\mathrm{st}}(x)$ is shifted toward the exit of the cell and the particles accumulate in front of the bottleneck. This behavior can also be observed in Fig. 5. In the large-force regime $P^{\mathrm{st}}(x)$ is almost constant over a wide range of $x$ values, indicating a minor influence of the shape of the structure on the dynamics of the particles. In the limit of large $f$ values the plateau extends and covers the whole period. In this situation, a deterministic treatment of the problem leads to adequate results.

\section{CONCLUSIONS}

With this work we have investigated the validity conditions under which the Fick-Jacobs approximation provides an accurate description of the biased diffusion of Brownian particles in 2D and 3D confined systems. We have established a validity criterion formulated in terms of the sinuosity of the channel $\omega(x)$, as was done in the unbiased case [21,24-26], and of the scaling parameter that causes forced diffusion. This scaling parameter compares the work done on a particle traveling a distance equal to the spatial period $L$ of the channel with the available thermal energy. Interestingly, the critical value of this scaling parameter up to which the Fick-Jacobs equation holds depends on the square of the period. This dependence follows from the analysis of the different time scales that rule the biased, diffusive dynamics. We have constructed a validity diagram showing the region of parameters (spanned by $f$ and $L$ ) in which the Fick-Jacobs approximation describes the overdamped diffusive transport accurately (Fig. 4). We have also investigated numerically the conditions for fast equilibration in the transverse direction, which is vital for the accuracy of the Fick-Jacobs approximation. The results presented evidence the usefulness of the Fick-Jacobs description with a spatial dependent diffusion coefficient at small applied bias. Obtaining a simplified Fick-Jacobs-type description that covers also intermediate to strong values of the scaled force still presents an open challenge. The availability of such a description would be beneficial for the detailed understanding of diffusive transport processes occurring in confined media, the latter dictating the nonequilibrium transport behavior in a great variety of systems far from thermal equilibrium [1-19,29,30].

\section{ACKNOWLEDGMENTS}

This work has been supported by the DGiCYT under Grant No. FIS2005-01299 (D.R.), the ESF STOCHDYN project (G.S., D.R., J.M.R., P.H.), the Alexander von Humboldt Foundation (J.M.R.), the Volkswagen Foundation under Project No. I/80424 (P.H.), the DFG collaborative research centre SFB-486, Project No. A10 (G.S., P.H.), the DFG Project No. 1517/26-1 (P.S.B., P.H.), and by the Nanosystems Initiative Munich (P.H.).

\section{APPENDIX: PARTICLE CURRENT}

The evolution equation of the probability distribution of overdamped Brownian particles in 1D can also be expressed as

$$
\frac{\partial P(x, t)}{\partial t}=\frac{\partial}{\partial x}\left(D(x) e^{-\beta A(x)} \frac{\partial}{\partial x} e^{\beta A(x)}\right) P(x, t),
$$

where $D(x)$ is a position-dependent diffusion coefficient, and $A(x)$ is the free energy landscape for the reaction coordinate $x$.

Equation (A1) results from the continuity equation

$$
\frac{\partial}{\partial t} P(x, t)=-\frac{\partial}{\partial x} J(x, t)
$$

with the probability current

$$
J(x, t)=-D(x) e^{-\beta A(x)} \frac{\partial}{\partial x} e^{\beta A(x)} P(x, t) .
$$

In the case of a periodic diffusion coefficient $D(x+L)$ $=D(x)$ and a tilted periodic free energy $A(x+L)=A(x)-F L$ it is convenient to define the reduced probability density and the corresponding current,

$$
\begin{gathered}
\hat{P}(x, t)=\sum_{n} P(n L+x, t), \\
\hat{J}(x, t)=\sum_{n} J(n L+x, t), \quad n \in \mathbb{Z} .
\end{gathered}
$$

By definition, these functions are periodic with periodicity $L, \quad \hat{P}(x+L, t)=\hat{P}(x, t)$ and $\hat{J}(x+L, t)=\hat{J}(x, t)$. Moreover, $\hat{P}(x, t)$ and $\hat{J}(x, t)$ enter the continuity equation (A2) and $\hat{P}(x, t)$ is normalized on any interval $(x, x+L)$, provided that $P(x, t)$ is normalized, e.g., $\int_{-\infty}^{+\infty} d x P(x, t)=1$. In the steadystate limit the probability current is a constant, $\hat{J}(x, t) \rightarrow \hat{J}$; thus Eq. (A3) becomes

$$
\hat{J}=-D(x) e^{-\beta A(x)} \frac{\partial}{\partial x} e^{\beta A(x)} \hat{P}^{\mathrm{st}}(x) .
$$


Multiplying both sides of Eq. (A6) by $1 / D(x) e^{\beta A(x)}$ and integrating over a period, we obtain

$$
\hat{J} \int_{x}^{x+L} \frac{1}{D\left(x^{\prime}\right)} e^{\beta A\left(x^{\prime}\right)} d x^{\prime}=\int_{x}^{x+L} \frac{\partial}{\partial x^{\prime}} e^{\beta A\left(x^{\prime}\right)} \hat{P}^{\mathrm{st}}\left(x^{\prime}\right) d x^{\prime} .
$$

Using now the conditions $\hat{P}^{\mathrm{st}}(x+L)=\hat{P}^{\mathrm{st}}(x)$ and $A(x+L)$ $=A(x)-\beta F L$, Eq. $(\mathrm{A} 7)$ reduces to

$$
\hat{J} \int_{x}^{x+L} \frac{1}{D\left(x^{\prime}\right)} e^{\beta A\left(x^{\prime}\right)} d x^{\prime}=\hat{P}^{\mathrm{st}}(x)\left(1-e^{-\beta F L}\right) e^{\beta A(x)} .
$$

Rearranging the terms and integrating over 0 to $L$, Eq. (A8) leads to

$\hat{J} \int_{0}^{L} e^{-\beta A(x)} d x \int_{x}^{x+L} \frac{1}{D\left(x^{\prime}\right)} e^{\beta A\left(x^{\prime}\right)} d x^{\prime}=\left(1-e^{-\beta F L}\right) \int_{0}^{L} \hat{P}^{\mathrm{st}}(x) d x$,

which due to the normalization yields

$$
\hat{J}=\frac{\left(1-e^{-\beta F L}\right)}{\int_{0}^{L} d x e^{-\beta A(x)} \int_{x}^{x+L} d x^{\prime} \frac{1}{D\left(x^{\prime}\right)} e^{\beta A\left(x^{\prime}\right)}} .
$$

The general relation between the stationary probability current and the steady-state particle current $(\langle\dot{x}\rangle)$ is

$$
\langle\dot{x}\rangle=\int_{0}^{L} \hat{J} d x,
$$

which implies $\langle\dot{x}\rangle=\hat{J} L$. Then the particle current reads

$$
\langle\dot{x}\rangle=\frac{\left(1-e^{-\beta F L}\right)}{\int_{0}^{L} \frac{d x}{L} e^{-\beta A(x)} \int_{x}^{x+L} d x^{\prime} \frac{1}{D\left(x^{\prime}\right)} e^{\beta A\left(x^{\prime}\right)} .}
$$

Remarkably, this expression for the particle current is equivalent to the expression obtained via the mean-firstpassage-time approach presented in Ref. [20].
[1] B. Hille, Ion Channels of Excitable Membranes (Sinauer, Sunderland, MA, 2001).

[2] R. M. Barrer, Zeolites and Clay Minerals as Sorbents and Molecular Sieves (Academic Press, London, 1978).

[3] T. Chou and D. Lohse, Phys. Rev. Lett. 82, 3552 (1999).

[4] L. Liu, P. Li, and S. A. Asher, Nature (London) 397, 141 (1999).

[5] Z. Siwy, I. D. Kosinska, A. Fulinski, and C. R. Martin, Phys. Rev. Lett. 94, 048102 (2005).

[6] A. M. Berezhkovskii and S. M. Bezrukov, Biophys. J. 88, L17 (2005).

[7] C. Kettner, P. Reimann, P. Hänggi, and F. Müller, Phys. Rev. E 61, 312 (2000).

[8] S. Matthias and F. Müller, Nature (London) 424, 53 (2003); F. Müller et al., Phys. Status Solidi A 182, 585 (2000).

[9] B.-Q. Ai and L.-G. Liu, Phys. Rev. E 74, 051114 (2006).

[10] P. Hänggi, F. Marchesoni, and F. Nori, Ann. Phys. 14, 51 (2005).

[11] R. D. Astumian and P. Hänggi, Phys. Today 55(11), 33 (2002).

[12] P. Reimann and P. Hänggi, Appl. Phys. A: Mater. Sci. Process. 75, 169 (2002).

[13] I. Derenyi and R. D. Astumian, Phys. Rev. E 58, 7781 (1998); T. A. J. Duke and R. H. Austin, Phys. Rev. Lett. 80, 1552 (1998); A. Van Oudenaarden and S. G. Boxer, Science 285, 1046 (1999); M. Kostur and L. Schimansky-Geier, Phys. Lett. A 265, 337 (2000); C. Keller, F. Marquardt, and C. Bruder, Phys. Rev. E 65, 041927 (2002).

[14] W. D. Volkmuth and R. H. Austin, Nature (London) 358, 600 (1992).

[15] G. I. Nixon and G. W. Slater, J. Chem. Phys. 117, 4042 (2002).

[16] R. Chang and A. Yethiraj, Phys. Rev. Lett. 96, 107802 (2006).
[17] U. Gerland, R. Bundschuh, and T. Hwa, Phys. Biol. 1, 19 (2004).

[18] R. Bundschuh and U. Gerland, Phys. Rev. Lett. 95, 208104 (2005).

[19] U. F. Keyser, B. N. Koeleman, S. Van Dorp, D. Krapf, R. M. M. Smeets, S. G. Lemay, N. H. Dekker, and C. Dekker, Nat. Phys. 2, 473 (2006).

[20] D. Reguera, G. Schmid, P. S. Burada, J. M. Rubí, P. Reimann, and P. Hänggi, Phys. Rev. Lett. 96, 130603 (2006).

[21] D. Reguera and J. M. Rubí, Phys. Rev. E 64, 061106 (2001).

[22] H. Risken, The Fokker-Planck Equation, 2nd ed. (Springer, Berlin, 1989).

[23] P. Hänggi and H. Thomas, Phys. Rep. 88, 207 (1982).

[24] M. H. Jacobs, Diffusion Processes (Springer, New York, 1967).

[25] R. Zwanzig, J. Phys. Chem. 96, 3926 (1992).

[26] P. Kalinay and J. K. Percus, Phys. Rev. E 74, 041203 (2006).

[27] R. L. Stratonovich, Radiotekh. Elektron. (Moscow) 3, 497 (1958); V. I. Tikhonov, Avtom. Telemekh. 20, 1188 (1959); R. L. Stratonovich, Topics in the Theory of Random Noise (Gordon and Breach, New York, 1967), Vol. II; Yu. M. Ivanchenko and L. A. Zil'berman, Zh. Eksp. Teor. Fiz. 55, 2395 (1968) [Sov. Phys. JETP 28, 1272 (1969)]; V. Ambegaokar and B. I. Halperin, Phys. Rev. Lett. 22, 1364 (1969).

[28] P. Hänggi, P. Talkner, and M. Borkovec, Rev. Mod. Phys. 62, 251 (1990).

[29] P. Reimann, C. Van den Broeck, H. Linke, P. Hänggi, J. M. Rubi, and A. Perez-Madrid, Phys. Rev. Lett. 87, 010602 (2001); P. Reimann, C. Van den Broeck, H. Linke, P. Hänggi, J. M. Rubi, and A. Perez-Madrid, Phys. Rev. E 65, 031104 (2002).

[30] E. Heinsalu, M. Patriarca, I. Goychuk, G. Schmid, and P. Hänggi, Phys. Rev. E 73, 046133 (2006). 